\title{
Endocarditis infecciosa cámaras derechas. Reporte de un caso
}

\author{
Case report: Right infectious endocarditis \\ Francisca Álvarez H. ${ }^{1}{ }^{*}$, Juan Torrez Z. ${ }^{1}$, Gustavo Galleguillos C. ${ }^{1}$, Joaquín Saavedra D. ${ }^{1}$ \\ 1 Hospital Guillermo Grant Benavente. Concepción, Chile.
}

Fecha de recepción: 23 de noviembre de 2020 / Fecha de aceptación: 20 de enero de 2021

\begin{abstract}
Infectious endocarditis (IE) of the right native valve refers to an infection in the tricuspid or pulmonary valves, whose mortality reaches $6 \%$. The main risk factors of IE are the intravenous drug use, the presence of intra-cardiac or vascular devices, and the underlying presence of cardiac malformations. Its nonspecific symptoms can lead to a late diagnosis with serious consequences, especially considering that the Duke criteria loses sensitivity in the right IE. Although the treatment is aimed at intravenous antimicrobial therapy it can also be surgical depending on the hemodynamic stability, complications, present pathogens and characteristics of the vegetation, among others. The case of a patient with risk factors who was diagnosed with right IE and whose treatment was surgical medical management, will be presented.
\end{abstract}

Key words: Right infectious endocarditis, tricuspid regurgitation.

\section{RESUMEN}

Endocarditis infecciosa (EI) de válvula nativa derecha hace referencia a una infección en las válvulas tricuspídea o pulmonar, cuya mortalidad alcanza el $6 \%$. Los principales factores de riesgo son el uso de drogas intravenosas, presencia de dispositivos intracardíacos o vasculares y la presencia subyacente de malformaciones cardiacas. Sus síntomas inespecíficos pueden llevar a un diagnóstico tardío con graves consecuencias, en especial considerando que los criterios de Duke pierden sensibilidad en El derecha. Si bien el tratamiento va dirigido a terapia antimicrobiana endovenosa, éste puede ser tanto médico como quirúrgico, según la estabilidad hemodinámica, complicaciones, patógenos presentes, características de las vegetaciones, entre otros. Se presentará el caso de una paciente con factores de riesgo que debuta con cuadro de El derecha llevándola a manejo médico quirúrgico.

Palabras clave: Endocarditis infecciosa derecha, insuficiencia tricuspídea.

\section{Presentación del caso}

Dechi aciente femenina, 31 años, venezolana con residencia en Chile desde el año 2018, alérgica a losartán y clembuterol, con antecedentes de Glomerulonefritis focal y segmentaria y trasplante a los 23 años de donante vivo. Luego de embarazo o a los 27 años, presenta rechazo renal, continuando con manejo médico. Evoluciona con hipertensión arterial secundaria y endocarditis bacteriana tricuspídea a los 29, tratada con antibioticoterapia. Finalmente, inicia hemodiálisis trisemanal a los 30 años.

Arriba a Chile el 20 de noviembre de 2018, luego de una semana sin hemodiálisis. Al tercer día consulta en el Servicio de Urgencias del Hospital de Curicó por edema y fiebre. Se pesqui- 
sa soplo tricuspídeo sistólico III/VI, por lo que se hospitaliza para manejo, estabilización y estudio.

Dentro de su estudio destaca: hemocultivos positivos para Streptococcus pneumoniae; angiotac de tórax negativo para tromboembolismo pulmonar; ecocardiograma transtorácico (ETT) con insuficiencia tricuspídea severa, perforación valva septal y presencia de 2 vegetaciones; dilatación de cavidades derechas y ventrículo izquierdo (VI) hipertrófico. Se decide traslado al Hospital Guillermo Grant Benavente de Concepción. Nuevo ETT concluye: VI de tamaño (DDV 51, DSV 33 mm) y fracción de eyección de $64 \%$, válvulas aórtica y mitral normales, válvula tricuspídea con vegetación de $8 \times 4 \mathrm{~mm}$, perforación del velo septal e insuficiencia severa, derrame pericárdico leve y presión sistólica de arteria pulmonar $51 \mathrm{mmHg}$.

Se programa cirugía para recambio valvular tricuspídeo biológico.

Evaluación preanestésica destaca Mallampati II, fístula arterio-venosa braquial izquierda, capacidad funcional (CF) III, sin otros hallazgos físicos de relevancia ni predictores de vía aérea difícil.

En pabellón, monitorización estándar ASA, con electrocardiografía continua 5 electrodos, presión arterial invasiva braquial izquierda, oximetría cerebral (NIRS), catéter venoso central yugular interno derecho para medición presión venosa central e introductor de Swan Ganz para medición de presión de arteria pulmonar en caso de necesidad de instalar su catéter.

Inducción con fentanilo, lidocaína, midazolam, propofol y rocuronio. Intubación orotraqueal tubo N 7,0 con balón, Cormark 1. Instalación de sonda ecocardiográfica transesofágica sin complicaciones y valoración de imágenes intraoperatorias (Figura 1). Mantención anestésica con sevofluorano al 1,5\% logrando MAC 1.0. Bombas de infusión continua de noradrenalina (4 mg/50 cc), dexmedetomidina (200 ug/50 cc) y nitroglicerina (50 mg/250 cc).

Se realiza esternotomía, pericardiotomía y se visualiza corazón aumentado de tamaño, contractilidad normal, sin observar cicatriz de infarto. Aorta no dilatada, sin placas palpables. Se administra heparina no fraccionada y se procede a canulación arterial aórtica ascendente, canulación venosa bicava e inicio de plegia anterógrada, entrando a circulación extracorpórea (CEC) en normotermia. Posterior a la atriotomía derecha, se logra observar una válvula tricuspídea con fibrosis, retracción y perforación del velo septal, con presencia de una vegetación de $5 \mathrm{~mm}$. Se reseca completamente la válvula y se implanta prótesis biológica mitral perimount $N^{\circ} 25$ en posición supra anular. Luego del cierre de la atriotomía y el desclampaje aórtico (22 minutos de clamp), el corazón retoma actividad eléctrica en bloqueo aurículo-ventricular completo (BAVC), debiendo ser apoyada por marcapasos transitorio. El ecocardiograma transesofágico (ETE) intraoperatorio muestra correcto funcionamiento de la prótesis. Salida de CEC sin problemas. Cierre parcial de pericardio, cierre esternal con puntos de alambre y cierre de piel intradérmico sin incidentes. Se traslada a Unidad de Cuidados Intensivos Coronarios (UCO), estable, con noradrenalina para PAM de 90 $\mathrm{mmHg}$ y $\mathrm{BIC}$ de propofol $2 \%$ para hipnosis.

En la UCO mantiene apoyo de marcapaso externo por BAVC, sin necesidad de drogas vasoactivas. Se extuba al día siguiente sin inconveniente, neurológicamente íntegra, glasgow 15 y hemodinamia autosostenida, con apoyo de marcapaso externo. Al séptimo día ingresa a pabellón y, bajo sedación, se implanta caja de marcapasos VVI, el cual inmediatamente inicia

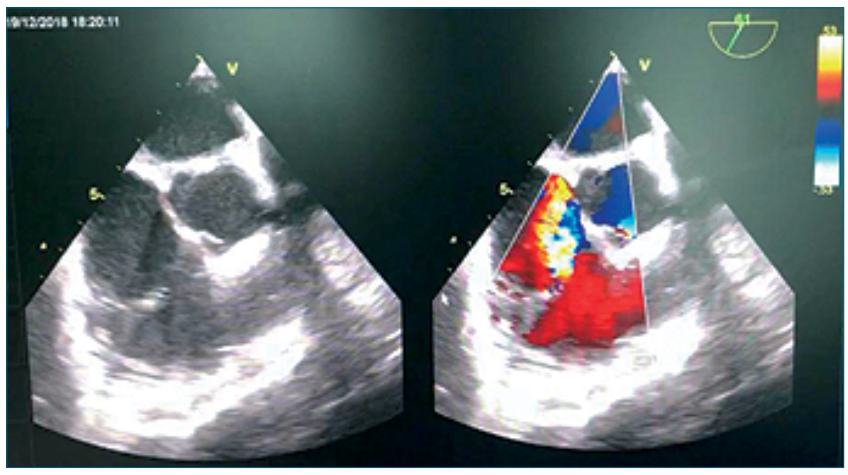

Figura 1. ME RV inflow-outflow: Se observa una extensa área de jet sobre AD.

un funcionamiento adecuado.

Evoluciona con hemodinamia estable, en ritmo marcapaso, manteniendo hemodiálisis trisemanal, por lo que se traslada a su hospital de origen para continuar con ceftriaxona por 14 días posrecambio valvular. Al completar el tratamiento, es dada de alta a su domicilio.

En control posoperatorio del 14 de enero de 2019, paciente en buen estado general, con disnea leve, con CF II, caminando cinco cuadras sin dificultades. Sin edema de extremidades ni soplo audible descrito previo a la cirugía. Es dada de alta para continuar manejo por equipo de Cardiología de su hospital de origen.

\section{Discusión}

Endocarditis infecciosa (EI) de válvula nativa derecha hace referencia a una infección en las válvulas tricuspídea (90\% de los casos) o pulmonar (10\%)[1]. Su mortalidad es el $6 \%$, siendo más letales aquellas con vegetaciones mayores de $2 \mathrm{~cm}$ o etiología micótica[2]. La El izquierda y derecha, simultánea, corresponde al $13 \%$, la derecha por sí sola al 10\%.

Los principales factores de riesgo de El tricuspídea son el uso de drogas intravenosas, presencia de dispositivos intracardíacos o vasculares, y la presencia subyacente de malformaciones cardiacas[3],[4],[5]. El 90\% de los pacientes con El derecha corresponden a personas con consumo de drogas inyectables; el $9 \%$ portadores de dispositivos vasculares y el $1 \%$ a personas con malformaciones cardiacas[4]. Cabe destacar el incremento de casos de endocarditis, viéndose reflejado en estudios recientes subiendo desde 0,2 a 2,7 hospitalizaciones por 100.000 habitantes entre los años 2010-2015 en EE.UU.[7] y entre ellos un aumento del $15 \%$ al $29 \%$ de casos correspondientes a usuarios de drogas entre los años 2010-2015[6].

En el ámbito nacional, existe un incremento de El derecha, siendo los principales factores de riesgo el uso de drogas intravenosas, y dispositivos cardíacos o vasculares, constituyendo la causa del caso presentado. Estudios chilenos muestran una incidencia de 2-3 casos por 100.000 habitantes/año de El "global"[15]. Mientras que en una revisión chilena del 2012-2016 la frecuencia de El derecha es de 3\% (tricúspide)[16].

El mecanismo de acción comienza con el daño endotelial causado en la válvula por material particulado, bacterias u hongos contenidos en drogas o jeringas para su uso, o en dispositi- 
vos intravasculares, contaminados al momento de la instalación o tras la manipulación de éstos[4],[8], para luego ser colonizado, siendo el patógeno más común el Staphylococcus aureus (70\% de los casos), seguidos por Streptococcus y enterococcus[9].

La fiebre es el síntoma más común, presente sobre el $90 \%$, acompañado de anorexia, pérdida de peso y malestar general. En el $75 \%$ de los casos de El tricuspídea, pueden generarse émbolos sépticos pulmonares que llevan a presencia de tos, disnea, dolor torácico y hemoptisis[3]. Si el diagnóstico es tardío, puede llevar a infartos pulmonares, abscesos, derrame pleural y empiema, pudiendo asociarse a falla cardiaca derecha, dilatación auricular y arritmias supraventriculares[1].

La sospecha debe establecerse en todo paciente con factores de riesgo que presenten fiebre y síntomas respiratorios, teniendo en cuenta que los criterios de Duke pierden sensibilidad en la El derecha, al carecer de la presentación clínica habitual de El izquierda[11],[12]. Para el estudio se procede a tomar hemocultivos y estudio ecocardiográfico transtorácico, y transesofágico en forma complementaria[10], siendo este último más sensible ( $90 \%$ vs $70 \%$ ), lo que permite visualizar vegetaciones de 1 a $2 \mathrm{~mm}$ de tamaño y complicaciones como insuficiencias valvulares, colecciones fistulosas intercavitarias y abscesos que lleven a inestabilidad hemodinámica[17].

El tratamiento va dirigido a terapia antimicrobiana endovenosa y retiro de dispositivos intravasculares causantes del cuadro. La resolución es quirúrgica en: vegetaciones sobre 20 mm, embolias pulmonares recurrentes, presencia de patógenos resistentes o bacteriemias persistentes, o insuficiencia tricuspídea severa[10],[13].

El manejo médico dependerá de la estabilidad hemodinámica del paciente, presencia de complicaciones estructurales y el patógeno detectado en los cultivos. Iniciando tratamiento antibiótico empírico en caso de pacientes hemodinámicamente inestables y esperando ajuste por resultado microbiológico cuando sea posible. En caso de Staphylococcus aureus meticilino sensibles, el tratamiento será con flucloxacilina o cloxacilina, alcanzando $89 \%$ de curación; o vancomicina en caso de pacientes con hipersensibilidad a betalactámicos[13],[14].

En el caso reportado, se evidencia la importancia de la sospecha clínica activa y dirigida de esta patología, especialmente en pacientes con factores de riesgo conocidos para un diagnóstico oportuno y precoz, llevando luego a un estudio adecuado, tanto microbiológico, a través de los hemocultivos, como imagenológico a través del ecocardiograma, sea transtorácico o transesofágico, que permitirán detectar la patología y las complicaciones secundarias a la misma. Cabe destacar la importancia de detectar precozmente complicaciones inicialmente inadvertidas, como son los émbolos sépticos pulmonares, los cuales fueron buscados dirigidamente a través de un AngioTAC de tórax. Finalmente, todo estudio debiese tener como finalidad cambios en la conducta. En este caso, los estudios deben llevar a la decisión terapéutica de tratamiento médico o quirúrgico y llevar el mejor manejo anestésico según requiera la valvulopatía correspondiente al paciente.

\section{Referencias}

1. Akinosoglou K, Apostolakis E, Marangos M, Pasvol G. Native valve right sided infective endocarditis. Eur J Intern Med 2013; 24:510.

2. Martín-Dávila P, Navas E, Fortún J, et al. Analysis of mortality and risk factors associated with native valve endocarditis in drug users: the importance of vegetation size. Am Heart J 2005; 150:1099.

3. Chahoud J, Sharif Yakan A, Saad H, Kanj SS. Right-Sided Infective Endocarditis and Pulmonary Infiltrates: An Update. Cardiol Rev 2016; 24:230.

4. Moreillon P, Que YA. Infective endocarditis. Lancet 2004; 363:139.

5. Smit J, Korup E, Schønheyder HC. Infections associated with permanent pacemakers and implanted cardioverter-defibrillator devices. A 10-year regional study in Denmark. Scand J Infect Dis 2010; 42:658.

6. Rudasill SE, Sanaiha Y, Mardock AL, et al. Clinical Outcomes of Infective Endocarditis in Injection Drug Users. J Am Coll Cardiol 2019; 73:559.

7. Fleischauer AT, Ruhl L, Rhea S, Barnes E. Hospitalizations for Endocarditis and Associated Health Care Costs Among Persons with Diagnosed Drug Dependence - North Carolina, 2010-2015. MMWR Morb Mortal Wkly Rep 2017; 66:569.

8. Weinstein WL, Brusch JL. Infective endocarditis, Oxford University Press, New York City 1996.

9. Mathew J, Addai T, Anand A, et al. Clinical features, site of involvement, bacteriologic findings, and outcome of infective endocarditis in intravenous drug users. Arch Intern Med 1995; 155:1641.

10. Daniel J Sexton, Vivian H Chu (2019). Right-sided native valve infective endocarditis. En Stephen B Calderwood (Ed), Ann Bolger (Ed), UpToDate. Recuperado el 2 de Abril del 2020, desde https://www.uptodate.com/contents/right-sided-native-valve-infective-endocarditis? search=right $\% 20$ side $\% 20$ endocarditis\&source=search_result\&selectedTitle=1 150\&usag e_type=default\&display_rank=1

11. Prendergast BD. Diagnostic criteria and problems in infective endocarditis. Heart 2004; 90:611.

12. Revilla A, López J, Villacorta E, et al. Isolated right-sided valvular endocarditis in non-intravenous drug users. Rev Esp Cardiol 2008; 61:1253.

13. Baddour LM, Wilson WR, Bayer AS, et al. Infective Endocarditis in Adults: Diagnosis, Antimicrobial Therapy, and Management of Complications: A Scientific Statement for Healthcare Professionals From the American Heart Association. Circulation 2015; 132:1435.

14. Ribera E, Gómez-Jimenez J, Cortes E, et al. Effectiveness of cloxacillin with and without gentamicin in short-term therapy for rightsided Staphylococcus aureus endocarditis. A randomized, controlled trial. Ann Intern Med 1996; 125:969.

15. Oyonarte M, Montagna R, Braun S, Maiers E, Rojo P, Cumsille JF. Infectious endocarditis: morbi-mortality in Chile. Results of the National Cooperative Study of Infective Endocarditis (1998-2002). Rev Med Chile 2003; 131 (3): 237-50.

16. Flores, Pablo et al. Endocarditis Infecciosa: caracterización clínica de la enfermedad. Revisión de casos de los últimos 5 años. Rev Chil Cardiol [online]. 2017, vol.36, n.1 [citado 2020-07-17], pp.34-40.

17. Nelson López Garzon, et al. Endocarditis por Staphylococcus aureus de la valvula tricuspidea posterior a un aborto septico: reporte de caso y revision de literatura.Rev Facultad Ciencias de la Salud. Universidad de Cauca. Vol 13 No. 2. Junio 2011. 